\title{
INCREASING OF ACCURACY FOR ENGINEERING CALCULATION OF HEAT TRANSFER PROBLEMS IN TWO LAYER MEDIA
}

H. KALIS ${ }^{1}$ and I. KANGRO ${ }^{2}$

${ }^{1}$ Institute of Mathematics Latvian Academy of Sciences and University of Latvia

Akadēmijas laukums 1, LV-1524 Rīga, Latvia

E-mail: kalis@lanet.lv

${ }^{2}$ Rēzekne Higher Education Institution, Departament of engineering science

Atbrīivoŝanas aleja 90, LV-4601, Rēzekne, Latvija

E-mail: kangro@cs.ru. lv

Received September 21, 2004; revised March 12, 2005

\begin{abstract}
In this paper we study the simple algorithms for modelling the heat transfer problem in two layer media. The initial model which is based on a partial differential equation is reduced to ordinary differential equations (ODEs). The increase of accuracy is shown if instead of first order ODE initial value problem $([4,5])$ the second order differential equations is taken. Such a procedure allows us to obtain a simple engineering algorithm for solving heat transfer equations in two layered domain of Cartesian, cylindrical (with axial symmetry) and spherical coordinates (with radial symmetry). In a stationary case the exact finite difference scheme is obtained.
\end{abstract}

Key words: heat transfer, layered media, numerical methods, mathematical modelling

\section{The Mathematical Model}

We shall consider the partial differential equations [4]:

$$
\gamma_{k} \frac{\partial u_{k}}{\partial t}=\frac{1}{p(x)} \frac{\partial}{\partial x}\left(\lambda_{k} p(x) \frac{\partial u_{k}}{\partial x}\right)-q_{k}(x, t)
$$

in multilayered domain $\Omega$ with $N$ layers

$$
\Omega=\left\{x: x \in\left[x_{k-1}, x_{k}\right], k=\overline{1, N}\right\}
$$

where $x_{0}=0, x_{N}=L, h_{k}=x_{k}-x_{k-1}, u_{k}=u_{k}(x, t)$ is the absolute temperature $[K]$ in the layer $\left[x_{k-1}, x_{k}\right], \gamma_{k}=c_{k} \rho_{k}, c_{k}\left[\frac{\mathrm{J}}{\mathrm{kg} \cdot \mathrm{K}}\right], \rho_{k}\left[\frac{\mathrm{kg}}{\mathrm{m}^{3}}\right], \lambda_{k}\left[\frac{\mathrm{W}}{\mathrm{m} \cdot \mathrm{K}}\right]$ are corresponding constants of specifi $\mathrm{c}$ thermal capacity, density and coeffi cients of heat 
conductivity in every layer, $t[s]$ is the time, $q_{k}=q_{k}(x, t)$ is the function of thermal sources, $x[m]$ is the space coordinate, $p=p(x)$ is given function depending on the system of coordinates: $p=1$ in the Cartesian coordinates, $p=x$ in cylindrical coordinates with an axial symmetry, $p=x^{2}$ in spherical coordinates with a radial symmetry.

Adding continuity conditions on surfaces $x=x_{k}, k=\overline{1, N-1}$

$$
\left\{\begin{array}{l}
u_{k}\left(x_{k}, t\right)=u_{k+1}\left(x_{k}, t\right) \\
\lambda_{k} \frac{\partial u_{k}\left(x_{k}, t\right)}{\partial x}=\lambda_{k+1} \frac{\partial u_{k+1}\left(x_{k}, t\right)}{\partial x},
\end{array}\right.
$$

boundary conditions on the surfaces $x=x_{0}=0, x=x_{n}=L$

$$
\left\{\begin{array}{l}
\lambda_{1} p(0) \frac{\partial u_{1}(0, t)}{\partial x}=\alpha_{0}\left(u_{1}(0, t)-T_{0}\right) \\
\lambda_{N} p(L) \frac{\partial u_{N}(L, t)}{\partial x}=f\left(u_{N}(L, t)\right)
\end{array}\right.
$$

and the initial condition at $t=0$

$$
u_{k}(x, t)=\phi(x), k=\overline{1, N}
$$

we obtain the initial-boundary value problem (1.1-1.4) for the heat transfer equation. The nonlinear function $f\left(u_{N}\right)$ in the boundary condition (1.3) describes the radiation from heaters and convection, for example

$$
f\left(u_{N}(L, t)\right)=\alpha_{L}\left(T_{L}-u_{N}(L, t)\right)+\epsilon \sigma\left(T_{*}^{4}-u_{N}^{4}(L, t)\right),
$$

where $\alpha_{0}, \alpha_{L}\left[\frac{W}{m^{3} . K}\right]$ are the coeffi cients of heat transfer, $\epsilon$ is the coeffi cient of emissivity $(\epsilon \in[0,1]), \sigma=5.6703 .10^{-8}\left[\frac{W}{m^{2} \cdot K^{4}}\right]$ is the Stefan-Boltzmann constant, $T_{0}, T_{L}, T_{*}$ are the constants of the temperatures outside the media and outside the heaters, $\phi=\phi(x)$ is the given initial temperature.

If $\alpha_{0}=\alpha_{L}=\infty$, then we have the fi rst kind boundary conditions in the form

$$
u_{1}(0, t)=T_{0}, \quad u_{N}(L, t)=T_{L} .
$$

If $p(0)=0$ then the fi rst boundary condition (1.3) is omitted and we can consider the symmetry condition

$$
\frac{\partial u_{1}(0, t)}{\partial x}=0
$$

In the case of homogeneous media we consider the following partial differential equation

$$
\frac{\partial u}{\partial t}=\frac{1}{p(x)} \frac{\partial}{\partial x}\left(p(x) \frac{\partial u}{\partial x}\right)-q(x, t),
$$

where the constants of heat transfer parameters $c, \rho, \lambda$ are normalizing magnitudes and $c \rho / \lambda$ is used as appropriate factor to the time $t$ and function $q$.

In every layer the heat equation (1.1) can be presented in the following form

$$
\frac{\partial}{\partial x}\left(\lambda_{k} \frac{\partial u_{k}(x, t)}{\partial x}\right)=F_{k}, \quad k=\overline{1, N},
$$

where $F_{k}=\gamma_{k} \dot{u}_{k}+q_{k}, \dot{u}_{k}=\frac{\partial u_{k}}{\partial t}$. 


\section{The Exact 3-Points Finite-Difference Scheme}

We use the method of fi nite volumes [3] for approximation of the differential problem. We consider $N+1$ grid points in the $x$ - direction

$$
0=x_{0}<x_{1}<\ldots<x_{N}=L .
$$

Then the exact fi nite-difference scheme for a given function $F_{k}$ is defi ned in the form [2]

$$
\begin{aligned}
& \lambda_{1} a_{1}\left(u_{1}-u_{0}\right)-\alpha_{0}\left(u_{0}-T_{0}\right)=\bar{R}_{0}^{+}, \\
& \lambda_{k+1} a_{k+1}\left(u_{k+1}-u_{k}\right)-\lambda_{k} a_{k}\left(u_{k}-u_{k-1}\right)=\bar{R}_{k}, k=\overline{1, N-1}, \\
& f\left(u_{N}\right)-\lambda_{N} a_{N}\left(u_{N}-u_{N-1}\right)=\bar{R}_{N}^{-},
\end{aligned}
$$

where

$$
\begin{aligned}
& \bar{R}_{k}=\bar{R}_{k}^{+}+\bar{R}_{k}^{-}, \quad \bar{R}_{k}^{+}=I_{k}^{+}+\gamma_{k+1} R_{k}^{+}, \quad \bar{R}_{k}^{-}=I_{k}^{-}+\gamma_{k} R_{k}^{-} \\
& I_{k}=I_{k}^{+}+I_{k}^{-}, \quad u_{k}=u_{k}(t)=u_{k}\left(x_{k}, t\right), k=\overline{1, N}, \quad u_{0}=u_{1}(0, t) \\
& R_{k}^{-}=\int_{x_{k-1}}^{x_{k}}\left(1-a_{k} \int_{x}^{x_{k}} \frac{d \psi}{p(\psi)}\right) p(x) \dot{u}_{k}(x, t) d x \\
& =a_{k} \int_{x_{k-1}}^{x_{k}}\left(p(x) \dot{u}_{k}(x, t) \int_{x_{k-1}}^{x} \frac{d \psi}{p(\psi)}\right) d x \\
& R_{k}^{+}=\int_{x_{k}}^{x_{k+1}}\left(1-a_{k+1} \int_{x_{k}}^{x} \frac{d \psi}{p(\psi)}\right) p(x) \dot{u}_{k+1}(x, t) d x \\
& =a_{k+1} \int_{x_{k}}^{x_{k+1}}\left(p(x) \dot{u}_{k+1}(x, t) \int_{x}^{x_{k+1}} \frac{d \psi}{p(\psi)}\right) d x \\
& a_{k}=\frac{1}{\int_{x_{k-1}}^{x_{k}} \frac{d x}{p(x)}}, \quad I_{k}^{+}=a_{k+1}^{x_{k+1}}\left(p(x) q_{x_{k}}(x, t) \int_{x}^{x_{k+1}} \frac{d \psi}{p(\psi)}\right) d x, \\
& I_{k}^{-}=\int_{x_{k-1}}^{x_{k}}\left(1-a_{k} \int_{x}^{x_{k}} \frac{d \psi}{p(\psi)}\right) p(x) q_{k}(x, t) d x \\
& =a_{k} \int_{x_{k-1}}^{x_{k}}\left(p(x) q_{k}(x, t) \int_{x_{k-1}}^{x} \frac{d \psi}{p(\psi)}\right) d x .
\end{aligned}
$$

If $p(0)=0$ (cylindrical and spherical coordinates), then $a_{1}=\alpha_{0}=0$ and the difference equation (2.1) is defi ned as

$$
\lambda_{1}\left(u_{1}-u_{0}\right)=R_{0}^{*},
$$

and the equation (2.2) for $k=1$ is given as

$$
\lambda_{2} a_{2}\left(u_{2}-u_{1}\right)=R_{1}^{*}
$$

where 


$$
\begin{aligned}
& R_{0}^{*}=\gamma_{1} \int_{0}^{x_{1}} p(x) \dot{u}_{1}(x, t) \int_{x}^{x_{1}} \frac{d \psi}{p(\psi)} d x+\int_{0}^{x_{1}} p(x) q_{1}(x, t) \int_{x}^{x_{1}} \frac{d \psi}{p(\psi)} d x \\
& R_{1}^{*}=\bar{R}_{1}^{+}+\gamma_{1} \int_{0}^{x_{1}} p(x) \dot{u}_{1}(x, t) d x+\int_{0}^{x_{1}} p(x) q_{1}(x, t) d x .
\end{aligned}
$$

Summarizing expressions (2.2), (2.3) we obtain equation

$$
f\left(u_{N}\right)=Q, \quad Q=\sum_{k=2}^{N-1} \bar{R}_{k}+\bar{R}_{N}^{-}+R_{0}^{*}+R_{1}^{*} .
$$

This equation has a positive root $u_{N}>0$, if $\epsilon \sigma T_{*}^{4}+\alpha_{L} T_{L}>Q$.

Next we consider only two layers, then $N=2, x_{1}=h_{1}, x_{2}=L=h_{1}+h_{2}$. Then the unknown values are $u_{1}, u_{2}\left(p(0)=1, \alpha_{0}=\infty\right)$ or $u_{0}, u_{1}, u_{2}(p(0)=0)$.

\section{The Cartesian Coordinates}

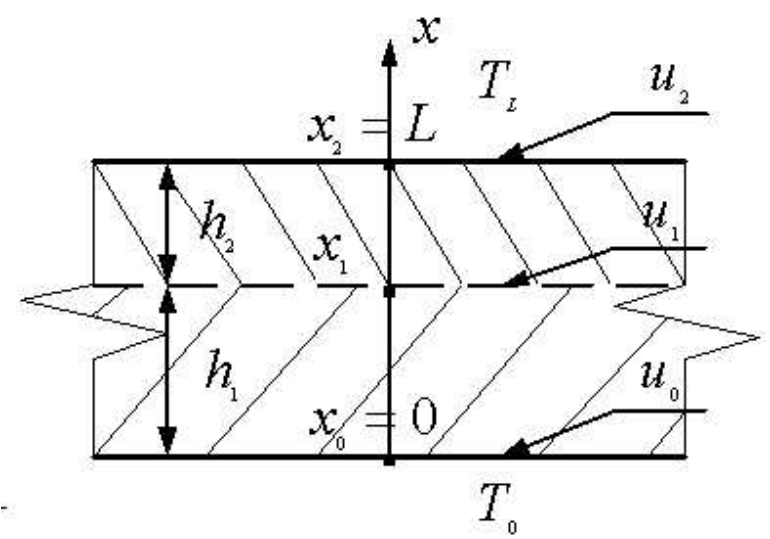

Figure 1. The calculated temperature $u_{1}$ in the layer $x=x_{1}$ in the case of Cartesian coordinates.

In this case (see fi g. 1)

$$
\begin{aligned}
& p(x)=1, \quad a_{k}=\frac{1}{h_{k}}, \quad R_{k}^{-}=\frac{1}{h_{k}} \int_{x_{k-1}}^{x_{k}}\left(x-x_{k-1}\right) \dot{u}_{k}(x, t) d x, \\
& R_{k}^{+}=\frac{1}{h_{k+1}} \int_{x_{k}}^{x_{k+1}}\left(x_{k+1}-x\right) \dot{u}_{k+1}(x, t) d x, \quad I_{k}^{-}=\frac{1}{h_{k}} \int_{x_{k-1}}^{x_{k}}\left(x-x_{k-1}\right) q_{k} d x, \\
& I_{k}^{+}=\frac{1}{h_{k+1}} \int_{x_{k}}^{x_{k+1}}\left(x_{k+1}-x\right) q_{k+1} d x,
\end{aligned}
$$


and the fi nite-difference scheme $\left(\alpha_{0}=\infty\right)$ is given in the form

$$
\left\{\begin{array}{l}
\frac{\lambda_{2}}{h_{2}}\left(u_{2}-u_{1}\right)-\frac{\lambda_{1}}{h_{1}}\left(u_{1}-T_{0}\right)=\gamma_{2} R_{1}^{+}+\gamma_{1} R_{1}^{-}+I_{1} \\
f\left(u_{2}\right)-\frac{\lambda_{2}}{h_{2}}\left(u_{2}-u_{1}\right)=\gamma_{2} R_{2}^{-}+I_{2}^{-} .
\end{array}\right.
$$

In the stationary case, if

$$
q_{k}=\text { const }, \quad \dot{u}_{k}=0, \quad I_{1}=0.5\left(q_{1} h_{1}+q_{2} h_{2}\right), \quad I_{2}=0.5 q_{2} h_{2},
$$

then

$$
f\left(u_{2}\right)+D_{12}\left(\left(T_{0}-u_{2}\right) D_{1}-Q_{12}\right)=Q_{2}, \quad u_{1}=\frac{D_{2} u_{2}+D_{1} T_{0}-Q_{12}}{D_{1}+D_{2}},
$$

where

$$
D_{i}=\frac{\lambda_{i}}{h_{i}}, \quad Q_{i}=0.5 q_{i} h_{i}, i=1,2, \quad D_{12}=\frac{D_{2}}{D_{1}+D_{2}}, \quad Q_{12}=Q_{1}+Q_{2} .
$$

Therefore the value $u_{2}$ can be obtained from the equation $u_{2}^{3}+a=\frac{b}{u_{2}}$, where

$$
a=\frac{\alpha_{L}+D_{12} D_{1}}{\epsilon \sigma}>0, \quad b=\frac{\epsilon \sigma T_{*}^{4}+\alpha_{L} T_{L}+D_{12} D_{1} T_{0}-Q_{2}-D_{12} Q_{12}}{\epsilon \sigma} .
$$

This equation have a positive root for $b>0$, e.g., $q_{1} \leq 0, q_{2} \leq 0$. The exact solution of the stationary problem (1.1)-(1.3) satisfi es expressions (3.2).

In the non-stationary case $\left(\dot{u}_{k} \neq 0\right)$, initial-value problems for ODE are used and we compute integrals $R_{i}^{ \pm}$approximately by the following quadrature formulas

$$
\begin{aligned}
& J_{m}=A_{1}^{(m)} V_{2}(0)+A_{2}^{(m)} V_{2}(1)+A_{3}^{(m)} V_{2}^{\prime}(1)+B_{2}^{(m)} V_{2}^{\prime \prime}(1)+r_{m}, m=1,2, \\
& J_{3}=A_{1}^{(3)} V_{1}(0)+A_{2}^{(3)} V_{1}(1)+A_{3}^{(3)} V_{1}^{\prime}(0)+B_{1}^{(3)} V_{1}^{\prime \prime}(0)+r_{3},
\end{aligned}
$$

where

$$
\begin{gathered}
J_{1}=\frac{R_{1}^{+}}{h_{2}}=\int_{0}^{1}(1-\bar{x}) V_{2}(\bar{x}) d \bar{x}, \quad J_{2}=\frac{R_{2}^{-}}{h_{2}}=\int_{0}^{1} \bar{x} V_{2}(\bar{x}) d \bar{x}, \\
J_{3}=\frac{R_{1}^{-}}{h_{1}}=\int_{0}^{1} \bar{x} V_{1}(\bar{x}) d \bar{x}, \quad V_{1}(\bar{x})=\dot{u}_{1}\left(\bar{x} h_{1}, t\right), \quad V_{2}(\bar{x})=\dot{u}_{2}\left(h_{1}+\bar{x} h_{2}, t\right), \\
r_{m}=\frac{h_{2}^{4}}{4 !} \frac{\partial^{4} \dot{u}\left(\xi_{m}, t\right)}{\partial x^{4}} C_{m}, \quad \xi_{m} \in(0, L),(m=1,2,3) \text { are the errors terms, } \\
A_{j}^{(m)}, B_{j}^{(m)}, C_{m},(j, m=1,2,3) \text { are the indefi nite coeffi cients. }
\end{gathered}
$$

Using the power functions $V_{j}(\bar{x})=\bar{x}^{i}, i=\overline{0,4}, j=1,2$ we get the systems of linear algebraic equations for $A_{j}^{(m)}, B_{j}^{(m)}$ :

$$
\left\{\begin{array}{l}
g(i, m)=A_{1}^{(m)} 0^{i}+A_{2}^{(m)}+i A_{3}^{(m)}+i(i-1) B_{2}^{(m)}, \\
\frac{1}{i+2}=A_{1}^{(3)} 0^{i}+A_{2}^{(3)}+i A_{3}^{(3)} 0^{i-1}+i(i-1) B_{1}^{(3)} 0^{i-2}
\end{array}\right.
$$


where

$$
m=1,2, \quad 0^{0}=1, \quad g(i, 1)=\frac{1}{(i+1)(i+2)}, \quad g(i, 2)=\frac{1}{i+2} .
$$

The solutions of these systems are given as

$$
\begin{aligned}
& A_{1}^{(1)}=\frac{1}{5}, \quad A_{2}^{(1)}=\frac{3}{10}, \quad A_{3}^{(1)}=-\frac{2}{15}, \quad B_{2}^{(1)}=\frac{1}{40} \\
& A_{1}^{(2)}=\frac{1}{20}, \quad A_{2}^{(2)}=\frac{9}{20}, \quad A_{3}^{(2)}=-\frac{7}{60}, \quad B_{2}^{(2)}=\frac{1}{60} \\
& A_{1}^{(3)}=\frac{3}{10}, \quad A_{2}^{(3)}=\frac{1}{5}, \quad A_{3}^{(3)}=\frac{2}{15}, \quad{ }_{1}^{(3)}=-\frac{1}{40}
\end{aligned}
$$

Constants $C_{m}$ in the residual $r_{m}$ are determined from (3.3) - (3.4), for $V_{1}(\bar{x})=$ $V_{2}(\bar{x})=\bar{x}^{4}: C_{1}=C_{3}=-\frac{1}{30}, C_{2}=-\frac{1}{60}$.

Using the difference equations (3.1) and the right hand side integrals approximating by (3.3), (3.4) with neglected error terms $r_{m}, m=1,2,3$, the approximate numerical solution $u_{1}(t), u_{2}(t)$ at every time step $t>0$ can be found by solving the following stiff system of two nonlinear ODEs of the second order

$$
\begin{aligned}
& \left(\dot{u}_{0}=\ddot{u}_{0}=0, \ddot{u}=\frac{\partial^{2}}{\partial t^{2}}, \alpha_{0}=\infty\right): \\
& \left\{\begin{array}{l}
\gamma_{2} h_{2}\left[A_{1}^{(1)} \dot{u}_{1}+A_{2}^{(1)} \dot{u}_{2}+\frac{h_{2}}{\lambda_{2}}\left(A_{3}^{(1)} f_{u}^{\prime}\left(u_{2}\right) \dot{u}_{2}+h_{2} B_{2}^{(1)}\left(\dot{q}_{2}(L, t)+\gamma_{2} \ddot{u}_{2}\right)\right)\right] \\
+\gamma_{1} h_{1}\left[A_{2}^{(3)} \dot{u}_{1}+\frac{h_{1}^{2}}{\lambda_{1}} B_{1}^{(3)} \dot{q}_{1}(0, t)\right]+I_{1}=\frac{\lambda_{2}}{h_{2}}\left(u_{2}-u_{1}\right)-\frac{\lambda_{1}}{h_{1}}\left(u_{1}-T_{0}\right),
\end{array}\right. \\
& \left\{\begin{array}{c}
\gamma_{2} h_{2}\left[A_{1}^{(2)} \dot{u}_{1}+A_{2}^{(2)} \dot{u}_{2}+\frac{h_{2}}{\lambda_{2}}\left(A_{3}^{(2)} f_{u}^{\prime}\left(u_{2}\right) \dot{u}_{2}\right.\right. \\
\left.\left.+h_{2} B_{2}^{(2)}\left(\dot{q}_{2}(L, t)+\gamma_{2} \ddot{u}_{2}\right)\right)\right]+I_{2}^{-}=f\left(u_{2}\right)-\frac{\lambda_{2}}{h_{2}}\left(u_{2}-u_{1}\right) .
\end{array}\right.
\end{aligned}
$$

Here one should take into account that from (1.1), (1.3) it follow that

$$
\begin{aligned}
V_{2}^{\prime}(1)=h_{2} \frac{\partial}{\partial x} \dot{u}_{2}(L, t)=h_{2} \frac{\partial}{\partial t} u_{2}^{\prime}(L, t) & =\frac{h_{2}}{\lambda_{2}} \dot{f}\left(u_{2}\right)=\frac{h_{2}}{\lambda_{2}} f_{u}^{\prime}\left(u_{2}\right) \dot{u}_{2}, \\
f_{u}^{\prime}\left(u_{2}\right) & =-\left(\alpha_{L}+4 \epsilon \sigma u_{2}^{3}\right), \quad V_{1}^{\prime}(0)=h_{1} \frac{\partial}{\partial x} \dot{u}_{1}(0, t)=h_{1} \frac{\partial}{\partial t} u_{1}^{\prime}(0, t)=h_{1} \alpha_{0} \dot{u}_{0}, \\
V_{2}^{\prime \prime}(1)=h_{2}^{2} \frac{\partial^{2}}{\partial x^{2}} \dot{u}(L, t)=h_{2}^{2} \frac{\partial}{\partial t} u^{\prime \prime}(L, t) & =\frac{h_{2}^{2}}{\lambda_{2}} \frac{\partial}{\partial t}\left(\gamma_{2} \dot{u}(L, t)+q(L, t)\right) \\
& =\frac{h_{2}^{2}}{\lambda_{2}}\left(\gamma_{2} \ddot{u}_{2}+\dot{q}_{2}\right) .
\end{aligned}
$$

The initial conditions for ODEs (3.6), (3.7) are the following

$$
\left\{\begin{array}{l}
u_{1}(0)=\phi\left(h_{1}\right), u_{2}(0)=\phi(L), \\
\dot{u}_{1}(0)=\left(\lambda_{1} \phi^{\prime \prime}\left(h_{1}\right)-q_{1}\left(h_{1}, 0\right)\right) / \gamma_{1}, \\
\dot{u}_{2}(0)=\left(\lambda_{2} \phi^{\prime \prime}(L)-q_{2}(L, 0)\right) / \gamma_{2} .
\end{array}\right.
$$


If the derivatives $\ddot{u}$ are not used in (3.3), (3.4) then [3]

$$
\left\{\begin{array}{l}
R_{k}^{-}=\gamma_{k} h_{k}\left(\frac{1}{6} \dot{u}_{k-1}+\frac{1}{3} \dot{u}_{k}-\frac{h_{k}^{2}}{24} \frac{\partial^{2} \dot{u}\left(\xi_{k}^{-}, t\right)}{\partial x^{2}}\right), \\
R_{k}^{+}=\gamma_{k+1} h_{k+1}\left(\frac{1}{3} \dot{u}_{k}+\frac{1}{6} \dot{u}_{k+1}-\frac{h_{k+1}^{2}}{24} \frac{\partial^{2} \dot{u}\left(\xi_{k}^{+}, t\right)}{\partial x^{2}}\right),
\end{array}\right.
$$

where $\xi_{k}^{-} \in\left(x_{k-1}, x_{k}\right), \xi_{k}^{+} \in\left(x_{k}, x_{k+1}\right)$.

For the equation (1.6), $h_{1}=h_{2}=h$ the fi nite difference scheme (3.1) is given in the form

$$
\left\{\begin{array}{l}
u_{2}-2 u_{1}+T_{0}=h\left(R_{1}+I_{1}\right) \\
h f\left(u_{2}\right)-u_{2}+u_{1}=h\left(R_{2}^{-}+I_{2}^{-}\right),
\end{array}\right.
$$

where

$$
\begin{gathered}
\frac{R_{1}}{h}=J_{1}=\int_{0}^{1} \bar{x} V(\bar{x}) d \bar{x}+\int_{1}^{2}(2-\bar{x}) V(\bar{x}) d \bar{x}, \\
\frac{R_{2}^{-}}{h}=J_{2}=\int_{1}^{2}(\bar{x}-1) V(\bar{x}) d \bar{x}, \quad V(\bar{x})=\dot{u}(\bar{x} h, t), \bar{x}=x / h, \\
\left\{\begin{array}{c}
J_{m}=A_{1}^{(m)} V(0)+A_{2}^{(m)} V(1)+A_{3}^{(m)} V(2)+A_{4}^{(m)} V^{\prime}(2) \\
+B_{1}^{(m)} V^{\prime \prime}(0)+B_{2}^{(m)} V^{\prime \prime}(1)+B_{3}^{(m)} V^{\prime \prime}(2)+r_{m},
\end{array}\right.
\end{gathered}
$$

$r_{m}=\frac{h^{7}}{7 !} \frac{\partial^{7} \dot{u}\left(\xi_{m}, t\right)}{\partial x^{7}} C_{m}, \xi_{m} \in(0, L), m=1,2$. Using power functions $V(\bar{x})=$ $\bar{x}^{i}, i=0, \ldots, 6$ in the expressions (3.11) we obtain two systems of 7 linear algebraic equations for determination of $A_{j}^{(m)}, B_{j}^{(m)}$

$$
\begin{aligned}
g(i, m)=A_{1}^{(m)} 0^{i} & +A_{2}^{(m)}+A_{3}^{(m)} 2^{i}+A_{4}^{(m)} i 2^{i-1} \\
& +i(i-1)\left(B_{1}^{(m)} 0^{i-2}+B_{2}^{(m)}+B_{3}^{(m)} 2^{i-2}\right), \quad m=1,2,
\end{aligned}
$$

where

$$
g(i, 1)=\frac{2^{i+2}-2}{(i+1)(i+2)}, \quad g(i, 2)=\frac{i 2^{i+1}+1}{(i+1)(i+2)} .
$$

Constants $C_{m}$ are determined from (3.11) for $V(\bar{x})=\bar{x}^{n}, n=7$ :

$$
\begin{aligned}
C_{m}=g(n, m)-A_{2}^{(m)} & -A_{3}^{(m)} 2^{n}-A_{4}^{(m)} n 2^{n-1} \\
& -n(n-1)\left(B_{2}^{(m)}+B_{3}^{(m)} 2^{n-2}\right) .
\end{aligned}
$$

The solution of system (3.12) is given by:

$$
\begin{aligned}
& A_{1}^{(1)}=A_{3}^{(1)}=\frac{11}{252}, \quad A_{2}^{(1)}=\frac{115}{126}, \quad A_{4}^{(1)}=0, \quad B_{2}^{(1)}=\frac{313}{7560}, \\
& B_{1}^{(1)}=B_{3}^{(1)}=-\frac{13}{15120}, \quad A_{1}^{(2)}=A_{3}^{(2)}=\frac{109}{504}, \quad A_{2}^{(2)}=\frac{269}{252}, \\
& A_{4}^{(2)}=0, \quad B_{1}^{(2)}=B_{3}^{(2)}=-\frac{83}{30240}, \quad B_{2}^{(2)}=\frac{1223}{15120} .
\end{aligned}
$$


We have from (3.13) that $C_{1}=0, C_{2}=\frac{32}{9}$. Therefore we get the residuals in the form $r_{1}=\frac{h^{8}}{8 !} \frac{\partial^{8} \dot{u}\left(\xi_{1}, t\right)}{\partial x^{8}} C_{1}$, and for $n=1$ we have from (3.13) $C_{1}=\frac{59}{1890}$.

$$
\text { If } B_{3}^{(1)}=B_{1}^{(1)}=0 \text {, then }
$$

$$
A_{1}^{(1)}=A_{3}^{(1)}=\frac{1}{30}, A_{2}^{(1)}=\frac{28}{30}, B_{2}^{(1)}=\frac{1}{20}, r_{1}=-\frac{1}{420} \frac{\partial^{6} \dot{u}(\xi, t)}{\partial x^{6}} h^{6}, \xi \in(0, L) .
$$

The system of ODEs (3.6)-(3.7) is presented in the form

$$
\begin{aligned}
h^{2}\left(A_{2}^{(1)} \dot{u}_{1}+A_{3}^{(1)} \dot{u}_{2}\right) & +h^{4}\left(B_{1}^{(1)} \dot{q}_{0}+B_{2}^{(1)}\left(\dot{q}_{1}+\ddot{u}_{1}\right)\right. \\
& \left.+B_{3}^{(1)}\left(\dot{q}_{2}+\ddot{u}_{2}\right)\right)+I_{1}=u_{2}-2 u_{1}+T_{0}, \\
h^{2}\left(A_{2}^{(2)} \dot{u}_{1}+A_{3}^{(2)} \dot{u}_{2}\right) & +h^{4}\left(B_{1}^{(2)} \dot{q}_{0}+B_{2}^{(2)}\left(\dot{q}_{1}+\ddot{u}_{1}\right)\right. \\
& \left.+B_{3}^{(2)}\left(\dot{q}_{2}+\ddot{u}_{2}\right)\right)+I_{2}^{-}=h f\left(u_{2}\right)-u_{2}+u_{1},
\end{aligned}
$$

where $\dot{q}_{j}=\dot{q}\left(x_{j}, t\right), j=0,1,2$. If the derivatives are not used in (3.11) then [3]

$$
A_{1}^{(1)}=A_{3}^{(1)}=\frac{1}{12}, \quad A_{2}^{(1)}=\frac{10}{12}, \quad r_{1}=\frac{h^{4}}{4 !} \frac{\partial^{4} \dot{u}(\xi, t)}{\partial x^{4}} C_{1}, \quad C_{1}=-\frac{1}{10} .
$$

Example 1. If

$$
q=0, \alpha_{L}=\infty, \phi(x)=\sin \left(\frac{\pi x}{L}\right), \dot{u}_{2}=\ddot{u}_{2}=0, T_{0}=T_{L}=0,
$$

then from the fi rst order ODE (3.14) with $A_{2}^{(1)}=\frac{5}{6}, B_{2}^{(1)}=0, u_{1}(0)=1$ it follows that $u_{1}(t)=\exp \left(-9.6 t / L^{2}\right)$. The exact solution of (1.6) is $u(x, t)=$ $\exp \left(-\pi^{2} t / L^{2}\right) \sin \left(\frac{\pi x}{L}\right)$ or $u_{1}=u(h, t)=\exp \left(-\pi^{2} t / L\right)$. Using the approximation

$$
u^{\prime \prime}(h, t) \approx \Lambda u_{1}=\frac{1}{h^{2}}\left(u_{0}(t)-2 u_{1}(t)+u_{2}(t)\right),
$$

we get thefi rst order ODE (the method of lines) $\dot{u}_{1}=\exp \left(-8 t / L^{2}\right)$. The second order ODE (3.14) is given as

$$
b_{1} \ddot{u}_{1}+a_{1} \dot{u}_{1}+u_{1}=0\left(b_{1}=h^{4} B_{2}^{(1)}, a_{1}=h^{2} A_{2}^{(1)}\right)
$$

with initial conditions $u_{1}(0)=1, \dot{u}_{1}(0)=\phi^{\prime \prime}(h)=-(\pi / L)^{2}$. It's solution is given by

$$
u_{1}(t)=D_{1} \exp \left(\mu_{1} t\right)+D_{2} \exp \left(\mu_{2} t\right),
$$

where $\mu_{1,2}=-a_{1} /\left(2 b_{1}\right) \pm \sqrt{\left(a_{1} /\left(2 b_{1}\right)\right)^{2}-1 / b_{1}}, D_{1}=\left(\mu_{2}+(\pi / L)^{2}\right) /\left(\mu_{2}-\mu_{1}\right)$, $D_{2}=-\left((\pi / L)^{2}+\mu_{1}\right) /\left(\mu_{2}-\mu_{1}\right)$.

Using the approximation $u^{\prime \prime}(h, t) \approx \Lambda u_{1}-\frac{h^{2}}{12} u^{(4)}(h, t), u^{(4)}(h, t)=\ddot{u}_{1}(t)$, we have the second order ODE with $b_{1}=\frac{h^{4}}{24}, a_{1}=\frac{h^{2}}{2}$ (the method of lines with a high order approximation).

The results of calculations are presented in Table 1. Here $L=2, u_{1 *}$ is the analytical solution, $u_{1 p p}$ is a $O\left(h^{8}\right)$ order approximation, $u_{1 p}$ is $O\left(h^{6}\right)$ order approximation, $u_{1}$ is a usual approximation, $u_{1 t}$ is obtained by using the method of lines, $u_{1 t t}$ is computed by the method of lines of high approximation. 
Increasing of Accuracy for Engineering Calculation of Heat Transfer Problems 181

Table 1. The values $u(1, t)$ at different time $t$ moments.

\begin{tabular}{ccccccc}
\hline$t$ & $u_{1 *}$ & $u_{1 p p}$ & $u_{1 p}$ & $u_{1}$ & $u_{1 t t}$ & $u_{1 t}$ \\
\hline .1 & .781344 & .781340 & .78127 & .7866 & .770 & .82 \\
.2 & .610498 & .610490 & .61032 & .6188 & .607 & .67 \\
.5 & .291213 & .291201 & .29094 & .3012 & .284 & .37 \\
.9 & .108537 & .108529 & .10834 & .1153 & .103 & .16 \\
\hline
\end{tabular}

\section{The Cylindrical Coordinates}

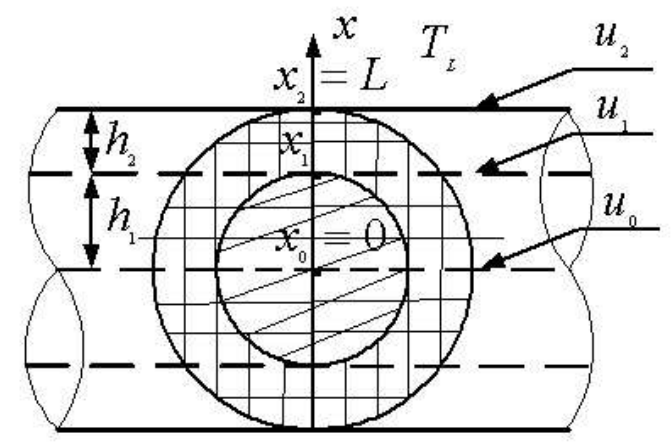

Figure 2. The calculated temperatures $u_{0}, u_{1}$ in layers $x_{0}=0$ and $x=x_{1}$ in the case of cylindrical coordinates.

In the cylindrical coordinates (see fi g.2) we get the following coeffi cients:

$$
\begin{aligned}
& p(x)=x, \quad a_{k}=\frac{1}{\ln \frac{x_{k}}{x_{k-1}}}, k \geq 2, \quad a_{1}=0, \\
& R_{k}^{-}=a_{k} \int_{x_{k-1}}^{x_{k}} x \ln \frac{x}{x_{k-1}} \dot{u}_{k}(x, t) d x, \quad R_{k}^{+}=a_{k+1} \int_{x_{k}}^{x_{k+1}} x \ln \frac{x_{k+1}}{x} \dot{u}_{k+1}(x, t) d x, \\
& I_{k}^{-}=a_{k} \int_{x_{k-1}}^{x_{k}} x \ln \frac{x}{x_{k-1}} q_{k} d x, \quad I_{k}^{+}=a_{k+1} \int_{x_{k}}^{x_{k+1}} x \ln \frac{x_{k+1}}{x} q_{k+1} d x .
\end{aligned}
$$

The fi nite difference scheme $\left(N=2, x_{1}=h_{1}, x_{2}=L=h_{1}+h_{2}, \beta=h_{2} / h_{1}\right)$ is given in the following form

$$
\left\{\begin{array}{l}
\lambda_{1}\left(u_{1}-u_{0}\right)=\gamma_{1} J_{2}+I_{0} \\
\lambda_{2} a_{2}\left(u_{2}-u_{1}\right)=\gamma_{2} R_{1}^{+}+I_{1}^{+} \gamma_{1} J_{1}+I_{0}^{(1)} \\
f\left(u_{2}\right)-\lambda_{2} a_{2}\left(u_{2}-u_{1}\right)=\gamma_{2} R_{2}^{-}+I_{2}^{-}
\end{array}\right.
$$

where 


$$
\begin{aligned}
& J_{2}=\int_{0}^{h_{1}} x \ln \frac{h_{1}}{x} \dot{u}_{1}(x, t) d x, \quad I_{0}=\int_{0}^{h_{1}} x \ln \frac{h_{1}}{x} q_{1}(x, t) d x, \\
& a_{2}=1 / \ln \frac{L}{h_{1}}, \quad J_{1}=\int_{0}^{h_{1}} x \dot{u}_{1}(x, t) d x, I_{0}^{(1)}=\int_{0}^{h_{1}} x q_{1}(x, t) d x .
\end{aligned}
$$

In the stationary case, if $q_{k}=$ const, $\dot{u}_{k}=0$, then

$$
\begin{aligned}
& I_{0}=\frac{q_{1} h_{1}^{2}}{4}, \quad I_{1}^{+}=q_{2}\left(-0.5 h_{1}^{2}+0.25\left(L^{2}-h_{1}^{2}\right) \ln ^{-1} \frac{L}{h_{1}}\right), \\
& I_{0}^{(1)}=\frac{q_{1} h_{1}^{2}}{2}, \quad I_{2}^{-}=q_{2}\left(0.5 L^{2}-0.25\left(L^{2}-h_{1}^{2}\right) \ln ^{-1} \frac{L}{h_{1}}\right)
\end{aligned}
$$

and the values $u_{0}, u_{1}, u_{2}$ can be obtained from the system of equations

$$
\left\{\begin{array}{l}
\lambda_{1}\left(u_{1}-u_{0}\right)=0.25 q_{1} h_{1}^{2} \\
\lambda_{2}\left(u_{2}-u_{1}\right)=0.5\left(q_{1}-q_{2}\right) h_{1}^{2} \ln \frac{L}{h_{1}}+0.25 q_{2}\left(L^{2}-h_{1}^{2}\right) \\
f\left(u_{2}\right) \ln \frac{L}{h_{1}}-\lambda_{2}\left(u_{2}-u_{1}\right)=q_{2}\left(0.5 L^{2} \ln \frac{L}{h_{1}}-0.25\left(L^{2}-h_{1}^{2}\right)\right)
\end{array}\right.
$$

The exact solution of the stationary problem (1.1)-(1.3) satisfi es expressions (4.2) and $f\left(u_{2}\right)=0.5\left(q_{2} L^{2}+\left(q_{1}-q_{2}\right) h_{1}^{2}\right)$. This equation has a positive root if

$$
\epsilon \sigma T_{*}^{4}+\alpha_{L} T_{L}-0.5\left(q_{2}\left(L^{2}-h_{1}^{2}\right)+q_{1} h_{1}^{2}\right)>0 .
$$

In the non-stationary case integrals $J_{1}, J_{2}, R_{1}^{+}, R_{2}^{-}$can be approximated by the following quadrature formulas

$$
\begin{aligned}
& \frac{J_{m}}{h_{1}^{2}}=A_{1}^{(m)} V_{1}(0)+A_{2}^{(m)} V_{1}(1)+A_{3}^{(m)} V_{1}^{\prime}(0)+B_{1}^{(m)} V_{1}^{\prime \prime}(0)+r_{m}, m=1,2 \\
& \frac{J_{m}}{h_{2}^{2}}=A_{1}^{(m)} V(0)+A_{2}^{(m)} V(1)+A_{3}^{(m)} V^{\prime}(1)+B_{2}^{(m)}\left(V^{\prime \prime}(1)+\frac{\beta V^{\prime}(1)}{1+\beta}\right)+r_{m}
\end{aligned}
$$

where $r_{m}=\frac{W^{(4)}\left(\xi_{m}\right)}{4 !} C_{m}, \xi_{m} \in(0,1), m=3,4 ; W=V_{1}, V$,

$V(\bar{x})=\dot{u}_{2}\left(h_{1}+h_{2} \bar{x}, t\right), \quad V_{1}(\bar{x})=u_{1}\left(h_{1} \bar{x}, t\right), \quad J_{1}=h_{1}^{2} \int_{0}^{1} \bar{x} V_{1}(\bar{x}) d \bar{x}$,

$J_{2}=-h_{1}^{2} \int_{0}^{1} \bar{x} \ln (\bar{x}) V_{1}(\bar{x}) d \bar{x}, \quad J_{3}=h_{2}^{2} \int_{0}^{1}\left(\beta^{-1}+\bar{x}\right) V(\bar{x}) \ln (1+\beta \bar{x}) d \bar{x}$,

$J_{4}=-h_{2}^{2} \int_{0}^{1}\left(\beta^{-1}+\bar{x}\right) V(\bar{x}) \ln \frac{1+\beta \bar{x}}{1+\beta} d \bar{x}, \quad R_{2}^{-}=\frac{J_{3}}{\ln (1+\beta)}, \quad R_{1}^{+}=\frac{J_{4}}{\ln (1+\beta)}$.

The unknown coeffi cients $A_{j}^{(m)}, B_{j}^{(m)}$ can be determinated by using $W(\bar{x})=\bar{x}^{i}, i=$ $0,1,2,3$, and solving the system of linear algebraic equations with parameter $\beta$ :

$$
\left\{\begin{array}{l}
g(i, m)=A_{1}^{(m)} 0^{i}+A_{2}^{(m)}+i A_{3}^{(m)} 0^{i-1}+i(i-1) B_{2}^{(m)} 0^{i-2}(m=1,2), \\
g(i, m)=A_{1}^{(m)} 0^{i}+A_{2}^{(m)}+i A_{3}^{(m)}+i(i-1+\beta /(1+\beta)) B_{2}^{(m)}(m=3,4),
\end{array}\right.
$$


where

$$
\begin{aligned}
& g(i, 1)=1 /(i+2), \quad g(i, 3)=\int_{0}^{1}\left(\beta^{-1}+\bar{x}\right) \ln (1+\beta \bar{x}) \bar{x}^{i} d \bar{x}, \\
& g(i, 2)=1 /(i+2)^{2}, \quad g(i, 4)=-\int_{0}^{1}\left(\beta^{-1}+\bar{x}\right) \ln \frac{1+\beta \bar{x}}{1+\beta} \bar{x}^{i} d \bar{x} .
\end{aligned}
$$

As an example, if $\beta=0.25$, then we get

$$
\begin{aligned}
& A_{1}^{(3)}=0.057368822, \quad A_{2}^{(3)}=0.5789255389, \quad A_{3}^{(3)}=-0.1486490492, \\
& B_{2}^{(3)}=0.02021926484, \quad C_{3}=-0.01752470, \\
& A_{1}^{(4)}=0.1505753322, \quad A_{2}^{(4)}=0.2528510778, \quad A_{3}^{(4)}=-0.1135916341, \\
& B_{2}^{(4)}=0.0202143207, \quad C_{4}=-0.03082307 .
\end{aligned}
$$

The other coeffi cients are given by

$$
\begin{aligned}
& A_{1}^{(1)}=\frac{3}{10}, \quad A_{2}^{(1)}=\frac{1}{5}, \quad A_{3}^{(1)}=\frac{2}{15}, \quad B_{1}^{(1)}=\frac{1}{40}, \quad C_{1}=-\frac{1}{30}, \\
& A_{1}^{(2)}=\frac{21}{100}, \quad A_{2}^{(2)}=\frac{1}{25}, \quad A_{3}^{(2)}=\frac{16}{225}, \quad B_{1}^{(2)}=\frac{9}{800}, \quad C_{2}=-\frac{11}{900} .
\end{aligned}
$$

The following stiff system of three ODEs of the second order is obtained for fi nding $u_{0}(t), u_{1}(t), u_{2}(t)$ :

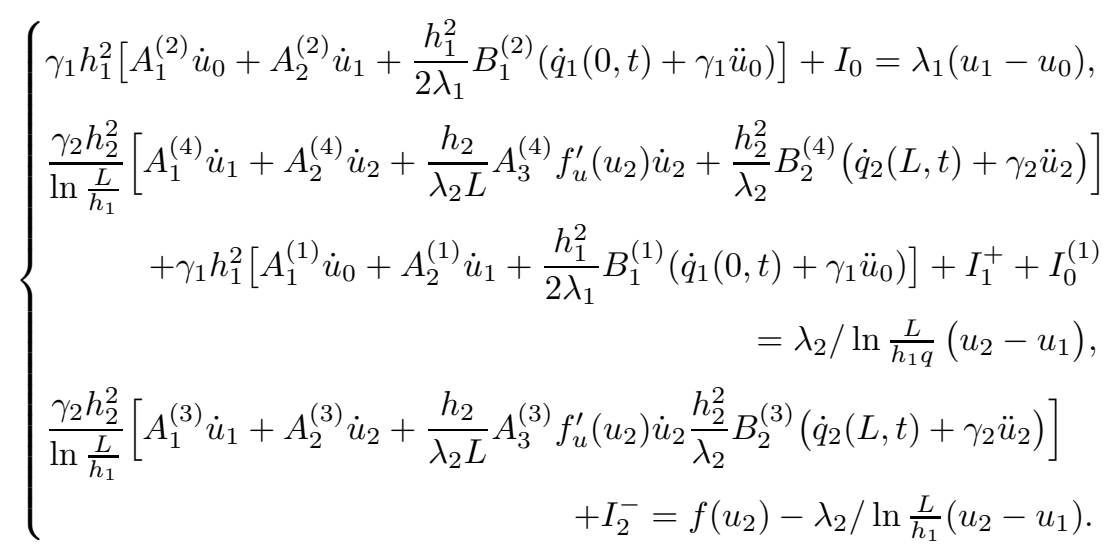

The initial conditions are

$$
\begin{gathered}
u_{0}(0)=\phi(0), \quad u_{1}(0)=\phi\left(h_{1}\right), \quad u_{2}(0)=\phi(L), \\
\dot{u}_{0}(0)=\left(2 \lambda_{1} \phi^{\prime \prime}(0)-q_{1}(0,0)\right) / \gamma_{1}, \\
\dot{u}_{2}(0)=\left(\lambda_{2}\left(\phi^{\prime \prime}(L)+L^{-1} \phi^{\prime}(L)\right)-q_{2}(L, 0)\right) / \gamma_{2} .
\end{gathered}
$$

For the equation (1.6) $\left(h_{1}=h_{2}=h=L / 2, \alpha_{L}=\infty\right)$ the fi nite difference scheme (4.1) is defi ned as 


$$
u_{1}-u_{0}=J_{2}+I_{0}, \quad T_{L}-u_{1}=J_{5}+I_{1}^{+}+I_{0}^{(1)},
$$

where $J_{5}=\ln 2 J_{1}+J_{0}^{*}, J_{0}^{*}=\int_{h}^{L} x \ln \frac{L}{x} \dot{u}(x, t) d x, V(\bar{x})=\dot{u}(\bar{x} h, t)$,

$$
\begin{aligned}
& \frac{J_{5}}{h^{2}}=\ln 2 \int_{0}^{2} \bar{x} V(\bar{x}) d \bar{x}-\int_{1}^{2} \bar{x} \ln \bar{x} V(\bar{x}) d \bar{x}=A_{1}^{(5)} V(0)+A_{2}^{(5)} V(1) \\
& +A_{3}^{(5)} V(2)+B_{1}^{(5)} V^{\prime \prime}(0)+\left.\frac{B_{2}^{(5)}\left(\bar{x} V^{\prime}(\bar{x})\right)^{\prime}}{\bar{x}}\right|_{\bar{x}=1}+\frac{V^{(5)}(\xi)}{5 !} C_{5}, \xi \in(0,1) .
\end{aligned}
$$

If $V(\bar{x})=\bar{x}^{i}, i=\overline{0,5}$, then we get the following results

$$
\begin{aligned}
& A_{1}^{(5)}=\frac{98}{2475}, \quad A_{2}^{(5)}=\frac{6653}{9900}, \quad A_{3}^{(5)}=\frac{19}{495} \\
& B_{1}^{(5)}=-\frac{53}{26400}, \quad B_{2}^{(5)}=\frac{287}{9900}, \quad C_{5}=-\frac{4028}{121275} .
\end{aligned}
$$

The following system of two ODEs of the second order is obtained for fi nding $u_{0}, u_{1}$ :

$$
\left\{\begin{array}{r}
h^{2}\left(A_{1}^{(2)} \dot{u}_{0}+A_{2}^{(2)} \dot{u}_{1}\right)+0.5 h^{4} B_{1}^{(2)}\left(\dot{q}_{0}+\ddot{u}_{0}\right)+I_{0}=u_{1}-u_{0} \\
h^{2}\left(A_{1}^{(5)} \dot{u}_{0}+A_{2}^{(5)} \dot{u}_{1}+A_{3}^{(5)} \dot{u}_{2}\right)+h^{4}\left(0.5 B_{1}^{(5)}\left(\dot{q}_{0}+\ddot{u}_{0}\right)+B_{2}^{(5)}\left(\dot{q}_{1}+\ddot{u}_{1}\right)\right) \\
+I_{1}^{+}+I_{0}^{(1)}=T_{L}-u_{1} .
\end{array}\right.
$$

Example 2. [5] If $q=0, T_{L}=0, \dot{u}_{2}=0, \phi(x)=J_{0}(\mu x / L)$ is the Bessel's function of first kind, $\mu=2.404825558$ the first positive root of equation $\oiint(\mu)=0$, then the exact solution is $u(x, t)=\exp \left(-(\mu / L)^{2} t\right) J_{0}(\mu x / L)$ with

$u_{0}(t)=\exp \left(-\left(\frac{\mu}{L}\right)^{2} t\right), u_{1}(t)=J_{0}\left(\frac{\mu}{2}\right) \exp \left(-\left(\frac{\mu}{L}\right)^{2} t\right), J_{0}\left(\frac{\mu}{2}\right)=0.6699297389$.

The results of calculations are presented in Table 2 , where $L=2, u_{0 *}, u_{1 *}$ are the exact values, $u_{0 p}, u_{1 p}$ approximate values.

Table 2. The values $u(0, t), u(1, t)$ in the time $t$.

\begin{tabular}{ccccc}
\hline$t$ & $u_{0 *}$ & $u_{0 p}$ & $u_{1 *}$ & $u_{1 p}$ \\
\hline .1 & .86538 & .86540 & .57974 & .57972 \\
.2 & .74889 & .74891 & .50171 & .50165 \\
.5 & .48534 & .48530 & .32515 & .32505 \\
.9 & .27220 & .27212 & .18236 & .18226 \\
\hline
\end{tabular}

Example 3. [1] For heat transfer in cylindrical wire-metal (copper) conductor $(x \in$ $\left.\left[0, h_{1}\right]\right)$ with insulation $\left(x \in\left(h_{1}, L\right]\right)$ the term $q_{1}$ describes Joule's heat generation 
and it can be written as [1] $q_{1}=-\sigma_{0}^{-1} \frac{I^{2}}{S^{2}}$, where $\sigma_{0}\left[\frac{1}{\Omega . m}\right]$ is the electric conductivity of metal, $I[A]$ is the electric current, $S\left[\mathrm{~m}^{2}\right]$ is the cross-section of the wire $\left(S=\pi h_{1}^{2}\right)$. The numerical results are obtained for the following values of parameters:

$$
\begin{aligned}
& h_{1}=4[\mathrm{~mm}], \quad L=5[\mathrm{~mm}], \quad \beta=0.25, \quad I=173[A], \quad \alpha_{L}=1\left[\frac{W}{\mathrm{~m}^{3} \mathrm{~K}}\right], \\
& \epsilon=0.5, \quad \sigma_{0}^{-1}=1.7 \cdot 10^{-8}[\Omega m], \quad c_{1}=410\left[\frac{\mathrm{J}}{\mathrm{kgK}}\right], \quad c_{2}=840\left[\frac{\mathrm{J}}{\mathrm{kgK}}\right], \\
& \rho_{1}=8960\left[\frac{\mathrm{kg}}{\mathrm{m}^{3}}\right], \quad \rho_{2}=500\left[\frac{\mathrm{kg}}{\mathrm{m}^{3}}\right], \quad q_{1}=-0.217 \cdot 10^{7}\left[\frac{\mathrm{W}}{\mathrm{m}^{3}}\right], \quad q_{2}=0, \\
& \lambda_{1}=400\left[\frac{\mathrm{W}}{\mathrm{mK}}\right], \quad \lambda_{2}=0.2\left[\frac{\mathrm{W}}{\mathrm{mK}}\right], \quad T_{*}=T_{L}=293[\mathrm{~K}] .
\end{aligned}
$$

We have calculated stationary and non-stationary solutions in two cases:

1) with radiation $u_{0}=316.78[K], u_{1}=316.76[K], u_{2}=297.42[K]$,

2) without radiation $u_{0}=329.69[K], u_{1}=329.67[K], u_{2}=310.33[K]$.

In the non-stationary case the results are presented in tables 3 (with radiation) and 4 (without radiation). We can see the effect of radiation.

Table 3. The values $u_{0}=u_{1}, u_{2}, \dot{u}_{0}, \dot{u}_{2}$ in time $t$ with radiation.

\begin{tabular}{lcccc}
\hline$t$ & $u_{0}$ & $\dot{u}_{0}$ & $u_{2}$ & $\dot{u}_{2}$ \\
\hline 1 & 293.6 & 0.560 & 293.1 & 0.094 \\
10 & 298.1 & 0.450 & 293.9 & 0.086 \\
50 & 309.7 & 0.171 & 296.1 & 0.032 \\
100 & 314.7 & 0.051 & 297.0 & 0.009 \\
150 & 316.2 & 0.015 & 297.3 & 0.003 \\
200 & 316.6 & 0.004 & 297.4 & 0.001 \\
\hline
\end{tabular}

Table 4. The values $u_{0}=u_{1}, u_{2}, \dot{u}_{0}, \dot{u}_{2}$ in time $t$ without radiation.

\begin{tabular}{lcccc}
\hline$t$ & $u_{0}$ & $\dot{u}_{0}$ & $u_{2}$ & $\dot{u}_{2}$ \\
\hline 1 & 293.6 & 0.560 & 293.1 & 0.193 \\
10 & 298.3 & 0.490 & 295.3 & 0.240 \\
50 & 312.8 & 0.260 & 302.3 & 0.132 \\
100 & 322.0 & 0.120 & 306.6 & 0.061 \\
150 & 326.1 & 0.055 & 308.6 & 0.026 \\
200 & 328.0 & 0.025 & 309.5 & 0.012 \\
\hline
\end{tabular}




\section{The Spherical Coordinates}

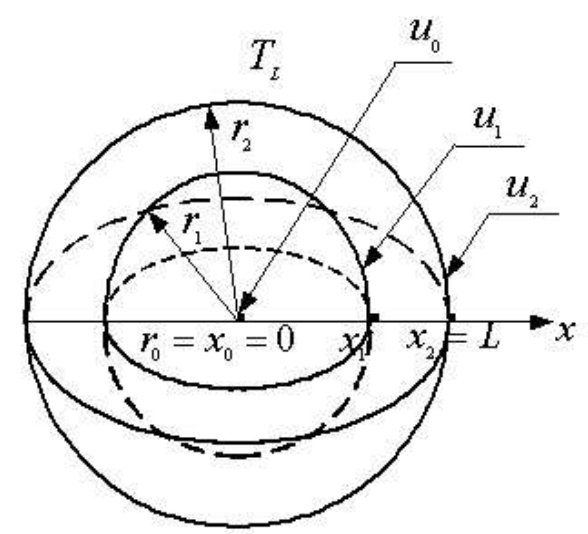

Figure 3. The calculated temperatures $u_{0}$ and $u_{1}$ in the points $x=0$ and $x=x_{1}$ in the case of spherical coordinates.

In the spherical coordinates (see Fig. 3) we get the following coeffi cients

$$
\begin{aligned}
& p(x)=x^{2}, \quad R_{k}^{-}=\frac{a_{k}}{x_{k-1}} \int_{x k-1}^{x_{k}} x\left(x-x_{k-1}\right) \dot{u}_{k}(x, t) d x, \\
& a_{k}=\left(\frac{1}{x_{k-1}}-\frac{1}{x_{k}}\right)^{-1}, \quad R_{k}^{+}=\frac{a_{k+1}}{x_{k+1}} \int_{x_{k}}^{x_{k+1}} x\left(x_{k+1}-x\right) \dot{u}_{k+1}(x, t) d x .
\end{aligned}
$$

The fi nite-difference scheme $(N=2)$ is given by (4.1), where

$$
\begin{aligned}
& J_{2}=h_{1}^{-1} \int_{0}^{h_{1}} x\left(h_{1}-x\right) \dot{u}_{1}(x, t) d x, \quad I_{0}=h_{1}^{-1} \int_{0}^{h_{1}} x\left(h_{1}-x\right) q_{1} d x, \\
& J_{1}=\int_{0}^{h_{1}} x^{2} \dot{u}_{1}(x, t) d x, \quad I_{0}^{(1)}=\int_{0}^{h_{1}} x^{2} q_{1} d x, \quad a_{2}=\frac{L}{\beta}, \beta=\frac{h_{2}}{h_{1}} .
\end{aligned}
$$

In the stationary case $\left(q_{k}=\right.$ const $)$ we get:

$$
\begin{aligned}
& I_{0}=\frac{q_{1} h_{1}^{2}}{6}, \quad I_{0}^{(1)}=\frac{q_{1} h_{1}^{3}}{3}, \\
& I_{2}^{-}=\frac{q_{2} L}{6 \beta}\left(2 \beta L^{2}+h_{1}^{2}-L^{2}\right), \quad I_{1}^{+}=\frac{q_{2}}{6 \beta}\left(L^{3}-h_{1}^{3}-3 \beta h_{1}^{3}\right) .
\end{aligned}
$$

From (4.1) it follows that

$$
\left\{\begin{array}{l}
\lambda_{1}\left(u_{1}-u_{0}\right)=\frac{q_{1} h_{1}^{2}}{6} \\
\frac{\lambda_{2} \beta}{L}\left(u_{2}-u_{1}\right)=\frac{q_{1} h_{1}^{3}}{3}+\frac{q_{2}}{6 \beta}\left(L^{3}-h_{1}^{3}-3 \beta h_{1}^{3}\right), \\
f\left(u_{2}\right)-\frac{\lambda_{2} \beta}{L}\left(u_{2}-u_{1}\right)=\frac{q_{2} L}{6 \beta}\left(2 \beta L^{2}+h_{1}^{2}-L^{2}\right) .
\end{array}\right.
$$


The exact solution of the problem (1.1)-(1.3) satisfi es (5.1) and

$$
f\left(u_{2}\right)-\frac{q_{2} L^{3}}{3}=\frac{h_{1}^{3}}{3}\left(q_{1}-q_{2}\right) .
$$

This equation has a positive root if

$$
\epsilon \sigma T_{*}^{4}+\alpha_{L} T_{L}-\frac{1}{3}\left(q_{2}\left(L^{3}-h_{1}^{3}\right)+q_{1} h_{1}^{3}\right)>0 .
$$

In the non-stationary case we have the following quadrature formulas $\left(x=\bar{x} h_{1}, x=\right.$ $\left.h_{1}+\bar{x} h_{2}\right)$ :

$\frac{J_{m}}{h_{1}^{3}}=A_{1}^{(m)} V_{1}(0)+A_{2}^{(m)} V_{1}(1)+A_{3}^{(m)} V_{1}^{\prime}(0)+B_{1}^{(m)} V_{1}^{\prime \prime}(0)+r_{m}, m=1,2$,

$\frac{J_{m}}{h_{2}^{2} L}=A_{1}^{(m)} V(0)+A_{2}^{(m)} V(1)+A_{3}^{(m)} V^{\prime}(1)+B_{2}^{(m)}\left(V^{\prime \prime}(1)+\frac{2 \beta V^{\prime}(1)}{1+\beta}\right)+r_{m}$,

where $r_{m}=\frac{W^{(4)}\left(\xi_{m}\right)}{4 !} C_{m}, \xi_{m} \in(0,1), m=3,4 ; W=V, V_{1}, m=1,2,3,4$,

$$
\begin{aligned}
& J_{1}=h_{1}^{3} \int_{0}^{1} \bar{x}^{2} V_{1}(\bar{x}) d \bar{x}, J_{2}=h_{1}^{3} \int_{0}^{1} \bar{x}(1-\bar{x}) V_{1}(\bar{x}) d \bar{x}, J_{3}=\beta R_{2}^{-}, J_{4}=\beta R_{1}^{+}, \\
& J_{3}=h_{2}^{2} L \int_{0}^{1}(\beta \bar{x}+1) V(\bar{x}) d \bar{x}, \quad J_{4}=\frac{h_{2}^{2} L}{1+\beta} \int_{0}^{1}(\beta \bar{x}+1)(1-\bar{x}) V(\bar{x}) d \bar{x} .
\end{aligned}
$$

The undefi nite coeffi cients are given by:

$$
\begin{aligned}
& A_{1}^{(1)}=A_{2}^{(1)}=\frac{1}{6}, \quad A_{3}^{(1)}=\frac{1}{12}, B_{1}^{(1)}=\frac{1}{60}, C_{1}=-\frac{1}{42}, A_{1}^{(2)}=\frac{2}{15}, \\
& A_{2}^{(2)}=\frac{1}{30}, \quad A_{3}^{(2)}=\frac{1}{20}, \quad B_{1}^{(2)}=\frac{1}{120}, \quad C_{2}=-\frac{1}{105}, A_{1}^{(3)}=\frac{\beta+3}{60}, \\
& A_{2}^{(3)}=\frac{19 \beta+27}{60}, \quad A_{3}^{(3)}=-\frac{5 \beta^{2}+13 \beta+7}{60(1+\beta)}, B_{2}^{(3)}=\frac{\beta+2}{120}, \quad C_{3}=-\frac{\beta+2}{120}, \\
& A_{1}^{(4)}=\frac{\beta+6}{30(1+\beta)}, \quad A_{2}^{(4)}=\frac{4 \beta+9}{30(\beta+1)}, A_{3}^{(4)}=-\frac{2 \beta^{2}+7 \beta+4}{30(1+\beta)^{2}}, \\
& B_{2}^{(4)}=\frac{\beta+3}{120(1+\beta)}, \quad C_{4}=-\frac{2 \beta+7}{210(1+\beta)} .
\end{aligned}
$$

Therefore we obtain the following system of ODEs: 


$$
\left\{\begin{array}{r}
\gamma_{1} h_{1}^{2}\left[A_{1}^{(2)} \dot{u}_{0}+A_{2}^{(2)} \dot{u}_{1}++\frac{h_{1}^{2} B_{1}^{(2)}}{3 \lambda_{1}}\left(\dot{q}_{1}(0, t)+\gamma_{1} \ddot{u}_{0}\right)\right]+I_{0}=\lambda_{1}\left(u_{1}-u_{0}\right), \\
\frac{\gamma_{2} h_{2}^{2} L}{\beta}\left[A_{1}^{(4)} \dot{u}_{1}+A_{2}^{(4)} \dot{u}_{2}+\frac{h_{2}}{\lambda_{2} L^{2}} A_{3}^{(4)} f_{u}^{\prime}\left(u_{2}\right) \dot{u}_{2}+\frac{h_{2}^{2}}{\lambda_{2}} B_{2}^{(4)}\left(\dot{q}_{2}(L, t)+\gamma_{2} \ddot{u}_{2}\right)\right] \\
+\gamma_{1} h_{1}^{3}\left[A_{1}^{(1)} \dot{u}_{0}+A_{2}^{(1)} \dot{u}_{1}+\frac{h_{1}^{2}}{3 \lambda_{1}} B_{1}^{(1)}\left(\dot{q}_{1}(0, t)+\gamma_{1} \ddot{u}_{0}\right)\right] \\
+I_{1}^{+}+I_{0}^{(1)}=\frac{\lambda_{2} L}{\beta}\left(u_{2}-u_{1}\right), \\
\frac{\gamma_{2} h_{2}^{2} L}{\beta}\left[A_{1}^{(3)} \dot{u}_{1}+A_{2}^{(3)} \dot{u}_{2}+\frac{h_{2}}{\lambda_{2} L^{2}} A_{3}^{(3)} f_{u}^{\prime}\left(u_{2}\right) \dot{u}_{2}+\frac{h_{2}^{2}}{\lambda_{2}} B_{2}^{(3)}\left(\dot{q}_{2}(L, t)+\gamma_{2} \ddot{u}_{2}\right)\right] \\
+I_{2}^{-}=f\left(u_{2}\right)-\frac{\lambda_{2} L}{\beta}\left(u_{2}-u_{1}\right) .
\end{array}\right.
$$

The initial conditions are given by

$$
\left\{\begin{array}{l}
u_{0}(0)=\phi(0), u_{1}(0)=\phi\left(h_{1}\right), u_{2}(0)=\phi(L), \\
\dot{u}_{0}(0)=\left(3 \lambda_{1} \phi^{\prime \prime}(0)-q_{1}(0,0)\right) / \gamma_{1}, \\
\dot{u}_{2}(0)=\left(\lambda_{2}\left(\phi^{\prime \prime}(L)+2 L^{-1} \phi^{\prime}(L)\right)-q_{2}(L, 0)\right) / \gamma_{2} .
\end{array}\right.
$$

For the equation (1.6) $\left(h_{1}=h_{2}=h=L / 2, \alpha_{L}=\infty, \dot{u}_{2}=\ddot{u}_{2}=0\right)$ we have the fi nite difference scheme in the form

$$
u_{1}-u_{0}=J_{2}+I_{0}, \quad T_{L}-u_{1}=J_{5}+I_{1}^{+}+I_{0}^{(1)},
$$

where

$$
\begin{aligned}
& J_{5}=\frac{J_{1}+J_{0}^{*}}{L}, \quad J_{0}^{*}=\int_{h}^{L} x(L-x) \dot{u}(x, t) d x, \\
& \frac{J_{5}}{h^{2}}=\frac{1}{2}\left(\int_{0}^{1} \bar{x}^{2} V(\bar{x}) d \bar{x}+\int_{1}^{2} \bar{x}(2-\bar{x}) V(\bar{x}) d \bar{x}\right)=A_{1}^{(5)} V(0)+A_{2}^{(5)} V(1) \\
& +A_{3}^{(5)} V(2)+B_{1}^{(5)} V^{\prime \prime}(0)+\left.\frac{B_{2}^{(5)}\left(\bar{x}^{2} V^{\prime}(\bar{x})\right)^{\prime}}{\bar{x}^{2}}\right|_{\bar{x}=1}+\left.\frac{B_{3}^{(5)}\left(\bar{x}^{2} V^{\prime}(\bar{x})\right)^{\prime}}{\bar{x}^{2}}\right|_{\bar{x}=2} \\
& \quad+\frac{V^{(6)}(\xi)}{6 !} C_{5}, \quad \xi \in(0,2), \quad V(\bar{x})=\dot{u}(\bar{x} h, t)=\bar{x}^{i}, i=0, \ldots, 6, \\
& A_{1}^{(5)}=\frac{1}{1008}, \quad A_{2}^{(5)}=\frac{115}{252}, \quad A_{3}^{(5)}=\frac{43}{1008}, \quad B_{1}^{(5)}=\frac{1}{5040}, \\
& B_{2}^{(5)}=\frac{107}{5040}, \quad B_{3}^{(5)}=-\frac{1}{1260}, \quad C_{5}=-\frac{1}{315} .
\end{aligned}
$$

If we add in expression $J_{5} / h^{2}$ the term $A_{4}^{(5)} V^{\prime}(0)$ with error term $\frac{V^{(7)}(\xi)}{7 !} C_{5}$, then we have the following values of coeffi cients:

$$
\begin{aligned}
& A_{1}^{(5)}=B_{1}^{(5)}=0, \quad A_{2}^{(5)}=\frac{115}{252}, \quad A_{3}^{(5)}=\frac{11}{252}, \quad B_{2}^{(5)}=\frac{313}{15120}, \\
& B_{3}^{(5)}=-B_{2}^{(5)}, \quad A_{4}^{(5)}=-B_{2}^{(5)}, \quad C_{5}=-\frac{59}{3780} .
\end{aligned}
$$




$$
\begin{gathered}
h^{2}\left(A_{1}^{(2)} \dot{u}_{0}+A_{2}^{(2)} \dot{u}_{1}\right)+h^{4}\left(B_{1}^{(2)} \frac{\dot{q}_{0}+\ddot{u}_{u}}{3}+B_{2}^{(2)}\left(\dot{q}_{1}+\ddot{u}_{1}\right)\right)+I_{0}=u_{1}-u_{0}, \\
h^{2}\left(A_{1}^{(5)} \dot{u}_{0}+A_{2}^{(5)} \dot{u}_{1}\right)+h^{4}\left(B_{1}^{(5)} \frac{\dot{q}_{0}+\ddot{u}_{0}}{3}+B_{2}^{(5)}\left(\dot{q}_{1}+\ddot{u}_{1}\right)+B_{3}^{(5)} \dot{q}_{2}\right) \\
+I_{1}^{+}+I_{0}^{(1)}=T_{L}-u_{1} .
\end{gathered}
$$

Example 4. If $q=0, T_{L}=0, \phi(x)=x^{-1} \sin (\pi x / L)$, then the exact solution is

$$
\begin{aligned}
& u(x, t)=\frac{1}{x} \exp \left(-(\pi / L)^{2} t\right) \sin (\pi x / L), \\
& u_{0}(t)=\frac{\pi}{L} \exp \left(-(\pi / L)^{2} t\right), \quad u_{1}(t)=\frac{2}{L} \exp \left(-(\pi / L)^{2} t\right) .
\end{aligned}
$$

We have solved the system (5.4)-(5.5) with initial conditions

$$
u_{0}(0)=\frac{\pi}{L}, \quad u_{1}(0)=\frac{2}{L}, \quad \dot{u}_{1}(0)=-\frac{2 \pi^{2}}{L^{3}}, \quad \dot{u}_{0}=-\left(\frac{\pi}{L}\right)^{3}, L=2 .
$$

The results are presented in Table 5 .

Table 5. The values $u(0, t), u(1, t)$ in the time $t$.

\begin{tabular}{ccccc} 
& $u_{0 *}$ & $u_{0 p}$ & $u_{1 *}$ & $u_{1 p}$ \\
\hline .1 & 1.22733 & 1.22741 & 0.781344 & 0.781339 \\
.2 & 0.95897 & 0.95907 & 0.610498 & 0.610488 \\
.5 & 0.45744 & 0.45749 & 0.291213 & 0.291198 \\
.9 & 0.17049 & 0.17050 & 0.108537 & 0.108527 \\
\hline
\end{tabular}

\section{Conclusions}

1. The proposed method allows us to reduce 1D heat transfer problem in Cartesian, cylindrical and spherical coordinates to the system of the ordinary differential equations of the second order.

2. The described methods make it possible to fi nd the distribution of temperature in the case of different layers with the heat source in between the layers and on layers borders.

3. In different coordinates it is possible to enlarge the accuracy of the given algorithm, when second order derivatives are used instead of fi rst order derivatives.

4. Such formulations have a big practical importance as compared to Cartesian coordinates, e.g. for analysis of heat transfer in cylindrical wire-metal (coper) conductor with insulation. 


\section{References}

[1] A. Ilgevičus and H.-D. Liess. Calculation of the heat transfer in cylindrical wires and electric fuses by implicit finite volume method. Mathematical Modelling and Analysis, 8(3), $217-228,2003$.

[2] H. Kalis. Effective finite-difference schemes for solving some heat transfer problems with convection in multilayer media. Int. Journ. of Heat and Mass Transfer, 43, 4467 - 4474, 2000.

[3] H. Kalis and I. Kangro. Simple algorithm's for the calculation of heat transport problem in plate. Mathematical Modelling and Analysis, 6(1), 85 - 96, 2001.

[4] H. Kalis and I. Kangro. Simple methods of engineering calculation for solving heat transfer problems. Mathematical Modelling and Analysis, 8(1), 33 - 42, 2003.

[5] H. Kalis and A. Lasis. Simple algorithm's for calculation the axial-symmetric heat transport problem in a cylinder. Mathematical Modelling and Analysis, 6(2), 262 - 269, 2001.

\section{Kai kurių inžinerinių, dvisluoksnėje srityje, šilumos laidumo uždavinių tikslumo didini- mas}

H. Kalis, I. Kangro

Šiame straipsnyje yra nagrinejjami paprasti dvisluoksnès srities šilumos laidumo problemos modeliavimo algoritmai, keičiant diferencialines lygtis dalinėmis išvestinėmis i paprastas diferencialines lygtis. Parodoma, kad didesnio tikslumo pasiekimui, vietoje pirmos eilès paprastų diferencialinių lygčių pradinio uždavinio nagrinèjamos antros eilès diferencialinès lyg-

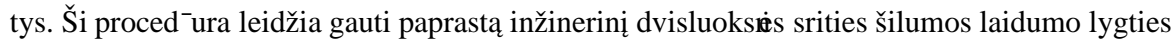
sprendini stačiakampèje, cilindrinèje (su ašių simetrija) ir sferinèje (su spinduline simetrija) koordinačių sistemoje. Tiksli baigtinių skirtumų schema buvo sudaryta stacionariam atvejui. 